\title{
Diagonalisierung des Hamilton-Operators in der feldtheoretischen Formulierung der nichtrelativistischen Mehrkanalstreutheorie*
}

\author{
R. WALTER \\ Institut für Theoretische Physik der Universität Stuttgart \\ (Z. Naturforsch. 25 a, 329—335 [1970]; eingegangen am 21. Dezember 1969)
}

\begin{abstract}
We discuss the general procedure of diagonalizing a field-theoretical Hamiltonian in the special case of a multi-channel scattering problem in which the asymptotic fields and the interpolating field are connected by a non-unitary transformation.
\end{abstract}

\section{Einleitung}

In der Mehrkanalstreutheorie treten im Gegensatz zur gewöhnlichen Streutheorie mehrere ein- und auslaufende Teilchensorten auf, die entweder aus einem oder aus Bindungszuständen mehrerer „elementarer" Teilchen bestehen. Wir wollen hier ein System von nichtrelativistischen Bose-Teilchen einer einzigen Sorte betrachten, in dem Bindungszustände auftreten.

WEIDLICH hat in einer früheren Arbeit ${ }^{1}$ gezeigt, daß zur Diagonalisierung des zugehörigen feldtheoretischen Hamilton-Operators, die ja einer Lösung der Schrödinger-Gleichung in allen $N$-Teilchenkonfigurationsräumen entspricht, im allgemeinen Fall der Anwesenheit von Bindungszuständen mehrere, sogen. asymptotische Felder einzuführen sind, die durch eine nicht-unitäre Transformation aus dem ursprünglichen, sogen. interpolierenden Feld zu konstruieren sind. Weidlich hat die nichtunitäre Transformation direkt durch die als bekannt vorausgesetzten Streulösungen der $N$-Teilchen-Schrödinger-Gleichung bestimmt.

In der vorliegenden Arbeit wollen wir dieses Problem mit einer anderen Methode angehen, die dem üblichen Vorgehen der sukzessiven Diagonalisierung des Hamilton-Operators in feldtheoretischen Problemstellungen entspricht. Wir erhalten für die explizit berechneten ersten Transformationskoeffizienten ein gekoppeltes, nichtlineares Integralgleichungssystem, das unter weiteren Nebenbedingungen gelöst werden muß. Die Lösung dieses Systems gelingt durch Vergleich mit der 2-Teilchen-Schrödinger-Gleichung. Die Nebenbedingungen entsprechen gerade der Vollständigkeits- und der Orthonormalitätsrela-

Sonderdruckanforderungen an Dr. Reinhold WALTER, Institut für Theoretische Physik der Universität Stuttgart, D-7000 Stuttgart 1, Azenbergstraße 12. tion der Lösungen der 2-Teilchen-Schrödinger-Gleichung. Ähnliche Untersuchungen lassen sich auch für die höheren Transformationskoeffizienten durchführen.

In $\S 1$ geben wir den Hamilton-Operator der nichtrelativistischen Mehrkanalstreutheorie in gewöhnlicher Formulierung als $N$-Teilchen-Schrödinger-Gleichung und in der feldtheoretischen Formulierung an und diskutieren einen allgemeinen Ansatz für eine nichtunitäre Transformation zur Diagonalisierung des feldtheoretischen Hamilton-Operators mit Hilfe von asymptotischen Feldern.

In $\S 2$ werden Bedingungen für die Transformationskoeffizienten untersucht. Wir erhalten für die explizit untersuchten ersten Transformationskoeffizienten ein gekoppeltes, nichtlineares Integralgleichungssystem, das in $\S 3$ durch Vergleich mit der 2-Teilchen-Schrödinger-Gleichung gelöst wird.

In § 4 untersuchen wir den Zusammenhang der Transformationskoeffizienten mit den gewöhnlichen Møller-Operatoren des 2-Teilchenproblems.

\section{§ 1. Hamilton-Operator der nichtrelativistischen Mehrkanalstreutheorie, Ansatz für nichtunitäre Transformation}

Wir betrachten $N$-Teilchensysteme, die durch die Schrödinger-Gleichung

$$
H^{(N)} \psi \pm^{(N)}(t)=i \dot{\psi} \pm^{(N)}(t) \quad(\hbar=1)
$$

mit

$$
H^{(N)}=H_{O}^{(N)}+V^{(N)}=\sum_{j=1}^{N}-\frac{1}{2 m} \Delta_{j}+\frac{1}{2} \sum_{i \neq j}^{N} V\left(\left|\boldsymbol{r}_{i}-\boldsymbol{r}_{j}\right|\right)
$$

beschrieben werden.

* Auszug aus der Dissertation, Universität Stuttgart 1969.

1 W. Weidlich, Z. Naturforsch. 18 a, 1266 [1963]. 
Der feldtheoretische Hamilton-Operator, der sämtliche $N$-Teilchensysteme von $N=0,1,2, \ldots$ zusammenfaßt, lautet

$$
\begin{aligned}
& H=\int \mathrm{d}^{3} \boldsymbol{k} \frac{\boldsymbol{k}^{2}}{2 m} \psi^{+}(\boldsymbol{k}) \psi(\boldsymbol{k}) \\
& +\frac{1}{2(2 \pi)^{3 / 2}} \int d^{3} \boldsymbol{k}_{1} \ldots \mathrm{d}^{3} \boldsymbol{k}_{4} \delta\left(\boldsymbol{k}_{1}+\boldsymbol{k}_{2}-\boldsymbol{k}_{3}-\boldsymbol{k}_{4}\right) \\
& \widetilde{V}\left(\left|\boldsymbol{k}_{2}-\boldsymbol{k}_{3}\right|\right) \times \psi^{+}\left(\boldsymbol{k}_{1}\right) \psi^{+}\left(\boldsymbol{k}_{2}\right) \psi\left(\boldsymbol{k}_{3}\right) \psi\left(\boldsymbol{k}_{4}\right),(1.3)
\end{aligned}
$$

wobei

$$
\widetilde{V}(|\boldsymbol{k}|)=\frac{1}{(2 \pi)^{3 / 2}} \int \mathrm{d}^{3} \boldsymbol{r} \exp \{i \boldsymbol{k} \cdot \boldsymbol{r}\} V(|\boldsymbol{r}|) .
$$

Die Teilchen sollen Bose-Charakter haben. Die Vernichtungs- bzw. Erzeugungsoperatoren $\psi(\boldsymbol{k})$ bzw. $\psi^{+}(\boldsymbol{k})$ der Teilchen mit Impuls $\boldsymbol{k}$ genügen dann den Vertauschungsrelationen

$$
\begin{aligned}
{\left[\psi(\boldsymbol{k}), \psi^{+}\left(\boldsymbol{k}^{\prime}\right)\right] } & =\delta\left(\boldsymbol{k}-\boldsymbol{k}^{\prime}\right), \\
{\left[\psi(\boldsymbol{k}), \psi\left(\boldsymbol{k}^{\prime}\right)\right] } & =0 .
\end{aligned}
$$

Der Hilbert-Raum $\boldsymbol{H}$ wird in üblicher Weise als Fock-Raum aus einem Vakum $\left|\Phi_{0}\right\rangle$ mit

$$
\psi(\boldsymbol{k})\left|\Phi_{0}\right\rangle=0 \quad \text { für alle } \boldsymbol{k}
$$

aufgebaut.

Nach den Untersuchungen von WeIDLICH ${ }^{1}$ kann im allgemeinen Fall der Anwesenheit einer Gruppe von 2-Teilchen-Bindungszuständen der feldtheoretische Hamilton-Operator, Gl. (1.3), durch eine nichtunitäre Transformation in die Diagonalform

$$
\begin{aligned}
H & =\int \mathrm{d}^{3} \boldsymbol{k} \frac{\boldsymbol{k}^{2}}{2 m} \boldsymbol{a}_{ \pm}^{+}(\boldsymbol{k}) \boldsymbol{a}_{ \pm}(\boldsymbol{k}) \\
& +\sum_{m} \int \mathrm{d}^{3} \boldsymbol{K}\left[\frac{\mathbf{K}^{2}}{4 m}-\varepsilon_{m}\right] b_{ \pm m}^{+}(\boldsymbol{K}) b_{ \pm m}(\boldsymbol{K})
\end{aligned}
$$

gebracht werden. Dabei sind $a_{ \pm}^{+}(\boldsymbol{k})$ bzw. $a_{ \pm}(\boldsymbol{k})$ Erzeugungs- bzw. Vernichtungsoperatoren für ein Teilchen mit Impuls $\boldsymbol{k}$, das sich für $t \rightarrow \pm \infty$ wie ein freies Teilchen bewegt, für endliche Zeiten aber in Wechselwirkung mit den anderen Teilchen steht. Weiter sind $b_{ \pm m}^{+}(\boldsymbol{K})$ bzw. $b_{ \pm m}(\boldsymbol{K})$ Erzeugungsbzw. Vernichtungsoperatoren für ein System aus zwei Teilchen mit Gesamtimpuls $\boldsymbol{K}$, das sich für $t \rightarrow \pm \infty$ wie ein freies 2-Teilchensystem im Bindungszustand $m$ verhält, für endliche Zeiten aber in Wechselwirkung mit den anderen Teilchen steht.

Die neuen Operatoren genügen den Vertauschungs. relationen

$\left[a_{ \pm}(\boldsymbol{k}), a_{ \pm}^{+}\left(\boldsymbol{k}^{\prime}\right)\right]=\delta\left(\boldsymbol{k}-\boldsymbol{k}^{\prime}\right)$,

$\left[a_{ \pm}(\boldsymbol{k}), a_{ \pm}\left(\boldsymbol{k}^{\prime}\right)\right]=0$,

$\left[b_{ \pm m}(\mathbf{K}), b_{ \pm m^{\prime}}^{+}\left(\boldsymbol{K}^{\prime}\right)\right]=\delta\left(\boldsymbol{K}-\boldsymbol{K}^{\prime}\right) \delta_{m, m^{\prime}}$,

$\left[b_{ \pm m}(\mathbf{K}), b_{ \pm m^{\prime}}\left(\boldsymbol{K}^{\prime}\right)\right]=0$,

$\left[a_{ \pm}(\boldsymbol{k}), b_{ \pm m}(\mathbf{K})\right]=\left[a_{ \pm}(\boldsymbol{k}), b_{ \pm m}^{+}(\boldsymbol{K})\right]=0$.

Der Hilbert-Raum $\boldsymbol{H}$ wird jetzt als Produktraum der Fock-Räume $\boldsymbol{H}_{a \pm}$ und $\boldsymbol{H}_{b \pm}$ gemäß

$$
\boldsymbol{H}=\boldsymbol{H}_{a \pm} \otimes \boldsymbol{H}_{b \pm}
$$

aufgebaut.

Auf Grund der Vertauschbarkeit des HamiltonOperators mit dem Teilchenzahloperator

$$
N=\int \mathrm{d}^{3} \boldsymbol{p} \psi^{+}(\boldsymbol{p}) \psi(\boldsymbol{p})
$$

lautet der allgemeine Ansatz für den Zusammenhang der Operatoren $\psi^{+}(\boldsymbol{k})$ bzw. $\psi(\boldsymbol{k})$ mit den Operatoren $\boldsymbol{a}_{ \pm}^{+}(\boldsymbol{k}), a_{ \pm}(\boldsymbol{k}), b_{ \pm m}^{+}(\boldsymbol{K})$ und $b_{ \pm m}(\boldsymbol{K})$

$$
\begin{aligned}
& \begin{aligned}
\psi^{+}(\boldsymbol{k}) & =\sum_{2 n+m} \sum_{m_{1} \ldots m_{n}} \sum_{m_{1}^{\prime} \ldots m^{\prime} n^{\prime}} \\
& =2 n^{\prime}+m^{\prime}+1
\end{aligned} \\
& \int \mathrm{d}^{3} \boldsymbol{K}_{1} \ldots \mathrm{d}^{3} \boldsymbol{K}_{n} \mathrm{~d}^{3} \boldsymbol{K}_{1}^{\prime} \ldots \mathrm{d}^{3} \boldsymbol{K}_{n^{\prime}}^{\prime} \mathrm{d}^{3} \boldsymbol{k}_{1} \ldots \mathrm{d}^{3} \boldsymbol{k}_{m} \mathrm{~d}^{3} \boldsymbol{k}_{1}{ }^{\prime} \ldots \mathrm{d}^{3} \boldsymbol{k}^{\prime}{ }_{m^{\prime}} \\
& \chi_{ \pm}\left(\boldsymbol{K}_{1}, m_{1} ; \ldots \boldsymbol{K}_{n}, m_{n}\left|\boldsymbol{k}_{1} \ldots \boldsymbol{k}_{m} \| \boldsymbol{K}_{1}{ }^{\prime}, m_{1}{ }^{\prime} ; \ldots \boldsymbol{K}_{n^{\prime}}, m_{n^{\prime}}{ }^{\prime}\right| \boldsymbol{k}_{1}{ }^{\prime}, \ldots \boldsymbol{k}_{m^{\prime}} ; \boldsymbol{k}\right) \\
& \times b_{ \pm m_{1}}^{+}\left(\boldsymbol{K}_{1}\right) \ldots b_{ \pm m_{n}}^{+}\left(\boldsymbol{K}_{n}\right) a_{ \pm}^{+}\left(\boldsymbol{k}_{1}\right) \ldots a_{ \pm}^{+}\left(\boldsymbol{k}_{m}\right) b_{ \pm m_{1}^{\prime}}\left(\boldsymbol{K}_{1}^{\prime}\right) \ldots b_{ \pm m^{\prime} n^{\prime}}\left(\boldsymbol{K}_{n^{\prime}}^{\prime}\right) a_{ \pm}\left(\boldsymbol{k}_{1}^{\prime}\right) \ldots a_{ \pm}\left(\boldsymbol{k}_{m^{\prime}}^{\prime}\right) \text {. }
\end{aligned}
$$

Bei Anwendung auf Räume mit beschränkter Teilchenzahl erhalten wir die Entwicklung

$$
\begin{aligned}
\psi^{+}(\boldsymbol{k}) & =\int \mathrm{d}^{3} \boldsymbol{k}_{1} \chi_{ \pm}\left(\boldsymbol{k}_{1} \| \boldsymbol{k}\right) a_{ \pm}^{+}\left(\boldsymbol{k}_{1}\right) \\
& +\int \mathrm{d}^{3} \boldsymbol{k}_{1} \mathrm{~d}^{3} \boldsymbol{k}_{2} \mathrm{~d}^{3} \boldsymbol{k}_{1}{ }^{\prime} \chi_{ \pm}\left(\boldsymbol{k}_{1}, \boldsymbol{k}_{2} \| \boldsymbol{k}_{1}{ }^{\prime} ; \boldsymbol{k}\right) a_{ \pm}^{+}\left(\boldsymbol{k}_{1}\right) a_{ \pm}^{+}\left(\boldsymbol{k}_{2}\right) \boldsymbol{a}_{ \pm}\left(\boldsymbol{k}_{1}{ }^{\prime}\right) \\
& +\sum_{m_{1}} \int \mathrm{d}^{3} \boldsymbol{K}_{1} \mathrm{~d}^{3} \boldsymbol{k}_{1}{ }^{\prime} \chi_{ \pm}\left(\boldsymbol{K}_{1}, m_{1} \| \boldsymbol{k}_{1}^{\prime} ; \boldsymbol{k}\right) b_{ \pm m_{1}}^{+}\left(\boldsymbol{K}_{1}\right) a_{ \pm}\left(\boldsymbol{k}_{1}{ }^{\prime}\right)+O(2) .
\end{aligned}
$$

Eine analoge Beziehung folgt für den hermitesch konjugierten Operator. Dabei bedeutet $O(N)$ die Reste, die nur Beiträge in $n$-Teilchenräumen mit $n \geqq N$ ergeben. 
Für den Koeffizienten $\chi_{ \pm}\left(\boldsymbol{k}_{1}, \boldsymbol{k}_{2} \| \boldsymbol{k}_{1}{ }^{\prime} ; \boldsymbol{k}\right)$ gilt auf gemäß

Grund des Ansatzes die Symmetrierelation

$\chi_{ \pm}\left(\boldsymbol{k}_{1}, \boldsymbol{k}_{2} \| \boldsymbol{k}_{1}^{\prime} ; \boldsymbol{k}\right)=\chi_{ \pm}\left(\boldsymbol{k}_{2}, \boldsymbol{k}_{1} \| \boldsymbol{k}_{1}{ }^{\prime} ; \boldsymbol{k}\right)$.

\section{§ 2. Bedingungen für die Transformations- koeffizienten}

Zur Festlegung der Koeffizienten $\chi_{ \pm}$sind zwei Forderungen zu erfüllen:

a) Gültigkeit der Vertauschungsrelationen,

b) Diagonalisierung des Hamilton-Operators.

Diese Forderungen legen die Transformation noch nicht vollständig fest. Insbesondere ergeben sich die beiden durch \pm gekennzeichneten Lösungen, die durch die unitäre $S$-Matrix des Mehrkanalsystems

$$
a_{-}(\boldsymbol{k})=S a_{+}(\boldsymbol{k}) S^{-1} ; b_{-m}(\boldsymbol{K})=S b_{+m}(\boldsymbol{K}) S^{-1}
$$

[vgl. Gl. (2.16) bei WEIDLICH ${ }^{1}$ ] zusammenhängen.

\section{a) Forderungen an die Transformationskoeffizienten} aus Gültigkeit der Vertauschungsrelationen

Der Transformationsansatz Gl. (1.12) soll bei Gültigkeit der Vertauschungsrelationen der Operatoren $\left\{a_{ \pm}^{+}, a_{ \pm} ; b_{ \pm}^{+}, b_{ \pm}\right\}$die Vertauschungsrelationen der Operatoren $\left\{\psi^{+}, \psi\right\}$ wahren.

Aus der Forderung

$$
\left[\psi^{+}(\boldsymbol{k}), \psi^{+}\left(\boldsymbol{k}^{\prime}\right)\right]=0
$$

folgt beim Einsetzen der Entwicklungen Gl. (1.13) und Normalordnung die Forderung

$$
\begin{aligned}
& \int \mathrm{d}^{3} \boldsymbol{k}_{1} \mathrm{~d}^{3} \boldsymbol{k}_{2} \boldsymbol{a}_{ \pm}^{+}\left(\boldsymbol{k}_{1}\right) a_{ \pm}^{+}\left(\boldsymbol{k}_{2}\right) \\
\times & \int \mathrm{d}^{3} \boldsymbol{k}_{3}\left[\chi_{ \pm}\left(\boldsymbol{k}_{3} \| \boldsymbol{k}^{\prime}\right) \chi_{ \pm}\left(\boldsymbol{k}_{1}, \boldsymbol{k}_{2} \| \boldsymbol{k}_{3} ; \boldsymbol{k}\right)-\chi_{ \pm}\left(\boldsymbol{k}_{3} \| \boldsymbol{k}\right) \chi_{ \pm}\left(\boldsymbol{k}_{1}, \boldsymbol{k}_{2} \| \boldsymbol{k}_{3} ; \boldsymbol{k}^{\prime}\right)\right] \\
+ & \sum_{m} \mathrm{~d}^{3} \boldsymbol{K} b_{ \pm m}^{+}(\boldsymbol{K}) \\
\times & \int \mathrm{d}^{3} \boldsymbol{k}_{3}\left[\chi_{ \pm}\left(\boldsymbol{k}_{3} \| \boldsymbol{k}^{\prime}\right) \chi_{ \pm}\left(\boldsymbol{K}, m \| \boldsymbol{k}_{3} ; \boldsymbol{k}\right)-\chi_{ \pm}\left(\boldsymbol{k}_{3} \| \boldsymbol{k}\right) \chi_{ \pm}\left(\boldsymbol{K}, m \| \boldsymbol{k}_{3} ; \boldsymbol{k}^{\prime}\right)\right]+O(1)=0 .
\end{aligned}
$$

Durch Koeffizientenvergleich erhalten wir die Forderungen

$$
\int \mathrm{d}^{3} \boldsymbol{k}_{3}\left[\chi_{ \pm}\left(\boldsymbol{k}_{3} \| \boldsymbol{k}^{\prime}\right) \chi_{ \pm}\left(\boldsymbol{k}_{1}, \boldsymbol{k}_{2} \| \boldsymbol{k}_{3} ; \boldsymbol{k}\right)-\chi_{ \pm}\left(\boldsymbol{k}_{3} \| \boldsymbol{k}\right) \chi_{ \pm}\left(\boldsymbol{k}_{1}, \boldsymbol{k}_{2} \| \boldsymbol{k}_{3} ; \boldsymbol{k}^{\prime}\right)\right]=0
$$

und

$$
\int \mathrm{d}^{3} \boldsymbol{k}_{3}\left[\chi_{ \pm}\left(\boldsymbol{k}_{3} \| \boldsymbol{k}^{\prime}\right) \chi_{ \pm}\left(\boldsymbol{K}, m \| \boldsymbol{k}_{3} ; \boldsymbol{k}\right)-\chi_{ \pm}\left(\boldsymbol{k}_{3} \| \boldsymbol{k}\right) \chi_{ \pm}\left(\boldsymbol{K}, m \| \boldsymbol{k}_{3} ; \boldsymbol{k}^{\prime}\right)\right]=0 .
$$

Weitere Forderungen an die höheren Transformationskoeffizienten würden wir bei Berücksichtigung von höheren Gliedern der Entwicklung in Gl. (1.13) erhalten.

Analog folgt aus der Forderung

die Bedingung

$$
\left[\psi(\boldsymbol{k}), \psi^{+}\left(\boldsymbol{k}^{\prime}\right)\right]=\delta\left(\boldsymbol{k}-\boldsymbol{k}^{\prime}\right)
$$

wobei

$$
\begin{aligned}
\delta\left(\boldsymbol{k}-\boldsymbol{k}^{\prime}\right) & =\int \mathrm{d}^{3} \boldsymbol{k}_{1} \chi_{ \pm}^{*}\left(\boldsymbol{k}_{1} \| \boldsymbol{k}\right) \chi_{ \pm}\left(\boldsymbol{k}_{1} \| \boldsymbol{k}^{\prime}\right) \\
& +\int \mathrm{d}^{3} \boldsymbol{k}_{1} \mathrm{~d}^{3} \boldsymbol{k}_{2} D_{ \pm}\left(\boldsymbol{k}, \boldsymbol{k}^{\prime} ; \boldsymbol{k}_{1}, \boldsymbol{k}_{2}\right) a_{ \pm}^{+}\left(\boldsymbol{k}_{1}\right) a_{ \pm}\left(\boldsymbol{k}_{2}\right)+O(2),
\end{aligned}
$$

$D_{ \pm}\left(\boldsymbol{k}, \boldsymbol{k}^{\prime} ; \boldsymbol{k}_{1}, \boldsymbol{k}_{2}\right)$

$\left.=2 \int \mathrm{d}^{3} \boldsymbol{k}_{3} \chi_{ \pm}^{*}\left(\boldsymbol{k}_{3} \| \boldsymbol{k}\right) \chi_{ \pm}\left(\boldsymbol{k}_{1}, \boldsymbol{k}_{3} \| \boldsymbol{k}_{2} ; \boldsymbol{k}^{\prime}\right)\right]+2 \int \mathrm{d}^{3} \boldsymbol{k}_{3} \chi_{ \pm}^{*}\left(\boldsymbol{k}_{2}, \boldsymbol{k}_{3} \| \boldsymbol{k}_{1} ; \boldsymbol{k}\right) \chi_{ \pm}\left(\boldsymbol{k}_{3} \| \boldsymbol{k}^{\prime}\right)$

$+2 \int \mathrm{d}^{3} \boldsymbol{k}_{3} \mathrm{~d}^{3} \boldsymbol{k}_{4} \chi_{ \pm}^{*}\left(\boldsymbol{k}_{3}, \boldsymbol{k}_{4} \| \boldsymbol{k}_{1} ; \boldsymbol{k}\right) \chi_{ \pm}\left(\boldsymbol{k}_{3}, \boldsymbol{k}_{\mathbf{4}} \| \boldsymbol{k}_{2} ; \boldsymbol{k}^{\prime}\right)+\sum_{m} \int \mathrm{d}^{3} \boldsymbol{K} \chi_{ \pm}^{*}\left(\boldsymbol{K}, m \| \boldsymbol{k}_{1} ; \boldsymbol{k}\right) \chi_{ \pm}\left(\boldsymbol{K}, m \| \boldsymbol{k}_{2} ; \boldsymbol{k}^{\prime}\right)$.

Durch Koeffizientenvergleich entstehen die Forderungen

$$
\int \mathrm{d}^{3} \boldsymbol{k}_{\mathbf{1}} \chi_{ \pm}^{*}\left(\boldsymbol{k}_{\mathbf{1}} \| \boldsymbol{k}\right) \chi_{ \pm}\left(\boldsymbol{k}_{\mathbf{1}} \| \boldsymbol{k}^{\prime}\right)=\delta\left(\boldsymbol{k}-\boldsymbol{k}^{\prime}\right) \quad \text { und } \quad D_{ \pm}\left(\boldsymbol{k}, \boldsymbol{k}^{\prime} ; \boldsymbol{k}_{\mathbf{1}}, \boldsymbol{k}_{\mathbf{2}}\right)=0 .
$$

\section{b) Forderungen an die Transformationskoeffizienten aus der Diagonalisierung des Hamilton-Operators}

Wir setzen nun die Entwicklungen Gl. (1.13) in den feldtheoretischen Hamilton-Operator Gl. (1.3) ein. Wir betrachten zunächst den Trivialfall der niedrigsten Näherung. Durch Vergleich mit der Diagonalform Gl. (1.8) erhalten wir die Forderung

$$
\int \frac{\mathrm{d}^{3} \boldsymbol{k} \boldsymbol{k}^{2}}{2 m} \int \mathrm{d}^{3} \boldsymbol{k}_{1} \mathrm{~d}^{3} \boldsymbol{k}_{2} \chi_{ \pm}\left(\boldsymbol{k}_{1} \| \boldsymbol{k}\right) \chi_{ \pm}^{*}\left(\boldsymbol{k}_{2} \| \boldsymbol{k}\right) a_{ \pm}^{+}\left(\boldsymbol{k}_{1}\right) a_{ \pm}\left(\boldsymbol{k}_{2}\right)=\int \mathrm{d}^{3} \boldsymbol{k} \frac{\boldsymbol{k}^{2}}{2 m} a_{ \pm}^{+}(\boldsymbol{k}) a_{ \pm}(\boldsymbol{k})+O(2) \text {. }
$$


Dies ist für den Ansatz

$$
\chi\left(\boldsymbol{k} \| \boldsymbol{k}^{\prime}\right)=\delta\left(\boldsymbol{k}-\boldsymbol{k}^{\prime}\right)
$$

erfüllt. Man könnte hier einen beliebigen, aber trivialen Phasenfaktor hinzufügen. Dieser Ansatz erfüllt auch die Forderung in Gl. (2.8).

Aus Gln. (2.3) und (2.4) folgt mit Gl. (2.11) in Ergänzung zu Gl. (1.14) die Symmetrierelation

$$
\begin{aligned}
& \chi_{ \pm}\left(\boldsymbol{k}_{1}, \boldsymbol{k}_{2} \| \boldsymbol{k}^{\prime}, \boldsymbol{k}\right)=\chi_{ \pm}\left(\boldsymbol{k}_{1}, \boldsymbol{k}_{2} \| \boldsymbol{k}, \boldsymbol{k}^{\prime}\right) \\
& \chi_{ \pm}\left(\boldsymbol{K}, m \| \boldsymbol{k}^{\prime}, \boldsymbol{k}\right)=\chi_{ \pm}\left(\boldsymbol{K}, m \| \boldsymbol{k}, \boldsymbol{k}^{\prime}\right) .
\end{aligned}
$$

Unter Benutzung von Gl. (2.11) und diesen Symmetrierelationen wird der feldtheoretische HamiltonOperator in Gl. (1.3) beim Einsetzen der Entwicklungen Gl. (1.13) in der nächsthöheren Näherung

$$
\begin{aligned}
H=\int \mathrm{d}^{3} \boldsymbol{k} & \frac{\boldsymbol{k}^{2}}{2 m} \boldsymbol{a}_{ \pm}^{+}(\boldsymbol{k}) a_{ \pm}(\boldsymbol{k}) \\
& +\int \mathrm{d}^{3} \boldsymbol{k}_{a} \mathrm{~d}^{3} \boldsymbol{k}_{b} \mathrm{~d}^{3} \boldsymbol{k}_{c} \mathrm{~d}^{3} \boldsymbol{k}_{d} A_{ \pm}\left(\boldsymbol{k}_{a}, \boldsymbol{k}_{b} \| \boldsymbol{k}_{c}, \boldsymbol{k}_{d}\right) a_{ \pm}^{+}\left(\boldsymbol{k}_{a}\right) a_{ \pm}^{+}\left(\boldsymbol{k}_{b}\right) a_{ \pm}\left(\boldsymbol{k}_{c}\right) a_{ \pm}\left(\boldsymbol{k}_{d}\right) \\
& +\left\{\sum_{m} \int \mathrm{d}^{3} \boldsymbol{K} \mathrm{d}^{3} \boldsymbol{k}_{a} \mathrm{~d}^{3} \boldsymbol{k}_{b} B_{ \pm}\left(\boldsymbol{k}_{a}, \boldsymbol{k}_{b} \| \boldsymbol{K}, m\right) a_{ \pm}^{+}\left(\boldsymbol{k}_{a}\right) a_{ \pm}^{+}\left(\boldsymbol{k}_{b}\right) b_{ \pm m}(\mathbf{K})+h \cdot k\right\} \\
& +\sum_{m_{a}, m_{b}} \mathrm{~d}^{3} \boldsymbol{K}_{a} \mathrm{~d}^{3} \boldsymbol{K}_{b} C_{ \pm}\left(\boldsymbol{K}_{a}, m_{a} \| \boldsymbol{K}_{b}, m_{b}\right) b_{ \pm m_{a}}^{+}\left(\boldsymbol{K}_{a}\right) b_{ \pm m_{b}}\left(\boldsymbol{K}_{b}\right)+O(3)
\end{aligned}
$$

mit

$$
\begin{aligned}
& 2 A_{ \pm}\left(\boldsymbol{k}_{a}, \boldsymbol{k}_{b} \| \boldsymbol{k}_{c}, \boldsymbol{k}_{d}\right) \\
& =\left(\frac{\boldsymbol{k}_{a}^{2}}{2 m}+\frac{\boldsymbol{k}_{b}^{2}}{2 m}\right) \chi_{ \pm}^{*}\left(\boldsymbol{k}_{c}, \boldsymbol{k}_{d} \| \boldsymbol{k}_{a}, \boldsymbol{k}_{b}\right)+\left(\frac{\boldsymbol{k}_{c}^{2}}{2 m}+\frac{\boldsymbol{k}_{d}^{2}}{2 m}\right) \chi_{ \pm}\left(\boldsymbol{k}_{a}, \boldsymbol{k}_{b} \| \boldsymbol{k}_{c}, \boldsymbol{k}_{d}\right) \\
& +\int \mathrm{d}^{3} \boldsymbol{k}_{1} \mathrm{~d}^{3} \boldsymbol{k}_{\mathbf{2}}\left(\frac{\boldsymbol{k}_{1}^{2}}{2 m}+\frac{\boldsymbol{k}_{2}^{2}}{2 m}\right) \chi_{ \pm}\left(\boldsymbol{k}_{a}, \boldsymbol{k}_{b} \| \boldsymbol{k}_{1}, \boldsymbol{k}_{2}\right) \chi_{ \pm}^{*}\left(\boldsymbol{k}_{c}, \boldsymbol{k}_{d} \| \boldsymbol{k}_{1}, \boldsymbol{k}_{2}\right) \\
& +\frac{1}{2(2 \pi)^{3 / 2}} \delta\left(\boldsymbol{k}_{a}+\boldsymbol{k}_{b}-\boldsymbol{k}_{c}-\boldsymbol{k}_{d}\right)\left[\tilde{V}\left(\left|\boldsymbol{k}_{a}-\boldsymbol{k}_{c}\right|\right)+\left[\tilde{V}\left(\left|\boldsymbol{k}_{b}-\boldsymbol{k}_{c}\right|\right)\right]\right. \\
& +\frac{1}{2(2 \pi)^{3 / 2}} \int \mathrm{d}^{3} \boldsymbol{k}_{1} \mathrm{~d}^{3} \boldsymbol{k}_{2} \delta\left(\boldsymbol{k}_{a}+\boldsymbol{k}_{b}-\boldsymbol{k}_{1}-\boldsymbol{k}_{2}\right)\left[\tilde{V}\left(\left|\boldsymbol{k}_{a}-\boldsymbol{k}_{1}\right|\right)+\tilde{V}\left(\left|\boldsymbol{k}_{b}-\boldsymbol{k}_{1}\right|\right)\right] \chi_{ \pm}^{*}\left(\boldsymbol{k}_{c}, \boldsymbol{k}_{d} \| \boldsymbol{k}_{1}, \boldsymbol{k}_{2}\right) \\
& +\frac{1}{2(2 \pi)^{3 / 2}} \int \mathrm{d}^{3} \boldsymbol{k}_{1} \mathrm{~d}^{3} \boldsymbol{k}_{2} \delta\left(\boldsymbol{k}_{1}+\boldsymbol{k}_{2}-\boldsymbol{k}_{c}-\boldsymbol{k}_{d}\right)\left[\tilde{V}\left(\left|\boldsymbol{k}_{1}-\boldsymbol{k}_{c}\right|\right)+\tilde{V}\left(\left|\boldsymbol{k}_{\mathbf{2}}-\boldsymbol{k}_{c}\right|\right)\right] \chi_{ \pm}\left(\boldsymbol{k}_{a}, \boldsymbol{k}_{b} \| \boldsymbol{k}_{1}, \boldsymbol{k}_{\mathbf{2}}\right) \\
& +\frac{1}{2(2 \pi)^{3 / 2}} \int \mathrm{d}^{3} \boldsymbol{k}_{1} \mathrm{~d}^{3} \boldsymbol{k}_{2} \mathrm{~d}^{3} \boldsymbol{k}_{3} \mathrm{~d}^{3} \boldsymbol{k}_{4} \delta\left(\boldsymbol{k}_{1}+\boldsymbol{k}_{2}-\boldsymbol{k}_{3}-\boldsymbol{k}_{4}\right)\left[\tilde{V}\left(\left|\boldsymbol{k}_{1}-\boldsymbol{k}_{3}\right|\right)+\tilde{V}\left(\left|\boldsymbol{k}_{2}-\boldsymbol{k}_{3}\right|\right)\right] \\
& 2 B_{ \pm}\left(\boldsymbol{k}_{a}, \boldsymbol{k}_{b} \| \boldsymbol{K}, m\right)=\left(\frac{\boldsymbol{k}_{a}^{2}}{2 m}+\frac{\boldsymbol{k}_{b}^{2}}{2 m}\right) \chi_{ \pm}^{*}\left(\boldsymbol{K}, m \| \boldsymbol{k}_{a}, \boldsymbol{k}_{b}\right) \\
& +\int \mathrm{d}^{3} \boldsymbol{k}_{1} \mathrm{~d}^{3} \boldsymbol{k}_{2}\left(\frac{\boldsymbol{k}_{1}^{2}}{2 m}+\frac{\boldsymbol{k}_{2}^{2}}{2 m}\right) \chi_{ \pm}\left(\boldsymbol{k}_{a}, \boldsymbol{k}_{b} \| \boldsymbol{k}_{1}, \boldsymbol{k}_{2}\right) \chi_{ \pm}^{*}\left(\boldsymbol{K}, m \| \boldsymbol{k}_{1}, \boldsymbol{k}_{\mathbf{2}}\right) \\
& +\frac{1}{2(2 \pi)^{3 / 2}} \int \mathrm{d}^{3} \boldsymbol{k}_{1} \mathrm{~d}^{3} \boldsymbol{k}_{2} \delta\left(\boldsymbol{k}_{a}+\boldsymbol{k}_{b}-\boldsymbol{k}_{1}-\boldsymbol{k}_{2}\right)\left[\tilde{V}\left(\left|\boldsymbol{k}_{a}-\boldsymbol{k}_{1}\right|\right)+\tilde{V}\left(\left|\boldsymbol{k}_{b}-\boldsymbol{k}_{1}\right|\right)\right] \chi_{ \pm}^{*}\left(\boldsymbol{K}, m \| \boldsymbol{k}_{1}, \boldsymbol{k}_{2}\right) \\
& +\frac{1}{2(2 \pi)^{3 / 2}} \int \mathrm{d}^{3} \boldsymbol{k}_{1} \mathrm{~d}^{3} \boldsymbol{k}_{2} \mathrm{~d}^{3} \boldsymbol{k}_{3} \mathrm{~d}^{3} \boldsymbol{k}_{4} \delta\left(\boldsymbol{k}_{1}+\boldsymbol{k}_{2}-\boldsymbol{k}_{3}-\boldsymbol{k}_{4}\right)\left[\tilde{V}\left(\left|\boldsymbol{k}_{1}-\boldsymbol{k}_{3}\right|\right)+\tilde{V}\left(\left|\boldsymbol{k}_{2}-\boldsymbol{k}_{3}\right|\right)\right] \\
& \times \chi_{ \pm}\left(\boldsymbol{k}_{a}, \boldsymbol{k}_{b} \| \boldsymbol{k}_{1}, \boldsymbol{k}_{2}\right) \chi_{ \pm}^{*}\left(\boldsymbol{K}, m \| \boldsymbol{k}_{3}, \boldsymbol{k}_{4}\right) ; \\
& 2 C_{ \pm}\left(\mathbf{K}_{a}, m_{a} \| \mathbf{K}_{b}, m_{b}\right) \\
& =\int \mathrm{d}^{3} \boldsymbol{k}_{1} \mathrm{~d}^{3} \boldsymbol{k}_{2}\left(\frac{\boldsymbol{k}_{1}^{2}}{2 m}+\frac{\boldsymbol{k}_{2}^{2}}{2 m}\right) \chi_{ \pm}\left(\boldsymbol{K}_{a}, m_{a} \| \boldsymbol{k}_{1}, \boldsymbol{k}_{2}\right) \chi_{ \pm}^{*}\left(\boldsymbol{K}_{b}, m_{b} \| \boldsymbol{k}_{1}, \boldsymbol{k}_{2}\right) \\
& +\frac{1}{2(2 \pi)^{3 / 2}} \int \mathrm{d}^{3} \boldsymbol{k}_{1} \mathrm{~d}^{3} \boldsymbol{k}_{2} \mathrm{~d}^{3} \boldsymbol{k}_{3} \mathrm{~d}^{3} \boldsymbol{k}_{4} \delta\left(\boldsymbol{k}_{1}+\boldsymbol{k}_{2}-\boldsymbol{k}_{3}-\boldsymbol{k}_{4}\right)\left[\tilde{V}\left(\left|\boldsymbol{k}_{1}-\boldsymbol{k}_{3}\right|\right)+\tilde{V}\left(\left|\boldsymbol{k}_{2}-\boldsymbol{k}_{3}\right|\right)\right] \\
& \times \chi_{ \pm}\left(\boldsymbol{K}_{a}, m_{a} \| \boldsymbol{k}_{1}, \boldsymbol{k}_{2}\right) \chi_{ \pm}^{*}\left(\boldsymbol{K}_{b}, m_{b} \| \boldsymbol{k}_{3}, \boldsymbol{k}_{4}\right) .
\end{aligned}
$$


Durch Vergleich mit der Diagonalform Gl. (1.8) des Hamilton-Operators erhalten wir die Forderungen

$$
\begin{gathered}
A_{ \pm}\left(\boldsymbol{k}_{a}, \boldsymbol{k}_{b} \| \boldsymbol{k}_{c}, \boldsymbol{k}_{d}\right)=0, \\
B_{ \pm}\left(\boldsymbol{k}_{a}, \boldsymbol{k}_{b} \| \mathbf{K}, m\right)=0, \\
C_{ \pm}\left(\boldsymbol{K}_{a}, m_{a} \| \boldsymbol{K}_{b}, m_{b}\right)=\left(\frac{\boldsymbol{k}_{a}^{2}}{4 m}-\varepsilon_{m_{a}}\right) \delta\left(\boldsymbol{K}_{a}-\boldsymbol{K}_{b}\right) \delta_{m_{a}, m_{b} .}
\end{gathered}
$$

Dies ist ein gekoppeltes, nichtlineares Integralgleichungssystem für die Transformationskoeffizienten

$$
\chi_{ \pm}\left(\boldsymbol{k}_{a}, \boldsymbol{k}_{b} \| \boldsymbol{k}_{c}, \boldsymbol{k}_{d}\right) \quad \text { und } \quad \chi_{ \pm}\left(\boldsymbol{K}_{a}, m_{a} \| \boldsymbol{k}_{c}, \boldsymbol{k}_{d}\right) .
$$

Die Integralgleichung (I) enthält nur die Transformationskoeffizienten $\chi_{ \pm}\left(\boldsymbol{k}_{a}, \boldsymbol{k}_{b} \| \boldsymbol{k}_{c}, \boldsymbol{k}_{d}\right)$, die Integralgleichung (III) nur die Koeffizienten $\chi_{ \pm}\left(\boldsymbol{K}_{a}, m_{a} \| \boldsymbol{k}_{c}, \boldsymbol{k}_{d}\right)$. Die homogene Integralgleichung (II) koppelt die beiden verschiedenen Typen von Transformationskoeffizienten.

Die Integralgleichung (II) ist für die Wahl $\chi_{ \pm}\left(\boldsymbol{K}^{\prime}, m^{\prime} \| \boldsymbol{k}_{c}, \boldsymbol{k}_{d}\right)=0$ eines bestimmten Bindungszustandes $\boldsymbol{K}^{\prime}, m^{\prime}$ erfüllt. Falls man die zugehörigen Operatoren $b_{ \pm m^{\prime}}^{+}\left(\boldsymbol{K}^{\prime}\right)$ und $b_{ \pm m^{\prime}}\left(\boldsymbol{K}^{\prime}\right)$ in dem Transformationsansatz Gl. (1.12) erst gar nicht einführt, ist auch die Integralgleichung (III) erfüllt. Die Integralgleichung (I) ist unabhängig von dieser Wahl erfüllbar. Dies bedeutet, daß wir auf Grund der Integralgleichungen (2.15) nicht gezwungen sind, alle Bindungszustände des betrachteten Systems in dem Transformationsansatz zu berücksichtigen.

Wir müssen neben den Integralgleichungen (2.15) aber auch noch die Bedingung (2.9) aus der Forderung nach Gültigkeit der Vertauschungsrelationen erfüllen. Wir werden in $\S 3$ [vgl. Gl. (3.7)] sehen, daß sich diese Bedingung gerade auf die Forderung nach Erfüllung der Vollständigkeitsrelation der 2-Teilchenzustände reduziert. Auf diese Weise wird die Berücksichtigung aller Bindungszustände des 2-Teilchensystems in dem Transformationsansatz erzwungen.

\section{§ 3. Lösung des Integralgleichungssystems}

Das Integralgleichungssystem (2.15) sieht zunächst recht kompliziert aus. Andererseits müßte dessen Lösung der Kenntnis aller 2-Teilchenzustände entsprechen, da wir bei der Entwicklung in Gl. (2.13) des Hamilton-Operators Glieder mit $O(3)$ vernachlässigt haben.

Führen wir mit der Transformation

mit

$$
\chi_{ \pm}\left(\boldsymbol{k}_{1}, \boldsymbol{k}_{2} \| \boldsymbol{k}_{1}{ }^{\prime}, \boldsymbol{k}_{2}{ }^{\prime}\right)=\chi_{ \pm}^{\prime}\left(\boldsymbol{k}_{1}, \boldsymbol{k}_{2} \| \boldsymbol{k}_{1}{ }^{\prime}, \boldsymbol{k}_{2}{ }^{\prime}\right)-\left[\delta\left(\boldsymbol{k}_{1}-\boldsymbol{k}_{1}{ }^{\prime}\right) \delta\left(\boldsymbol{k}_{2}-\boldsymbol{k}_{2}{ }^{\prime}\right)\right]_{\mathrm{sym} .}
$$

$$
\left[\delta\left(\boldsymbol{k}_{1}-\boldsymbol{k}_{1}{ }^{\prime}\right) \delta\left(\boldsymbol{k}_{2}-\boldsymbol{k}_{2}{ }^{\prime}\right)\right]_{\mathrm{sym} .} \equiv \frac{1}{2}\left[\delta\left(\boldsymbol{k}_{1}-\boldsymbol{k}_{1}{ }^{\prime}\right) \delta\left(\boldsymbol{k}_{2}-\boldsymbol{k}_{2}{ }^{\prime}\right)+\delta\left(\boldsymbol{k}_{2}-\boldsymbol{k}_{1}{ }^{\prime}\right) \delta\left(\boldsymbol{k}_{1}-\boldsymbol{k}_{2}{ }^{\prime}\right)\right]
$$

neue Koeffizienten $\chi_{ \pm}^{\prime}$ ein (für diese gelten die gleichen Symmetrierelationen wie für die Koeffizienten $\chi_{ \pm}$), so vereinfacht sich das Integralgleichungssystem beträchtlich, und durch geschickte Umformungen erhalten wir die Darstellung

(I)

$$
\begin{array}{r}
\times\left[\left(\frac{\boldsymbol{k}_{1}^{2}}{2 m}+\frac{\boldsymbol{k}_{2}^{2}}{2 m}\right) \chi_{ \pm}^{* *}\left(\boldsymbol{k}_{c}, \boldsymbol{k}_{d} \| \boldsymbol{k}_{1}, \boldsymbol{k}_{2}\right)+\frac{1}{(2 \pi)^{3 / 2}} \int \mathrm{d}^{3} \boldsymbol{k} \tilde{V}(|\boldsymbol{k}|) \chi_{ \pm}^{*}\left(\boldsymbol{k}_{c}, \boldsymbol{k}_{d} \| \boldsymbol{k}_{1}+\boldsymbol{k}, \boldsymbol{k}_{2}-\boldsymbol{k}\right)\right] \\
=\left(\frac{\boldsymbol{k}_{c}^{2}}{2 m}+\frac{\boldsymbol{k}_{d}^{2}}{2 m}\right)\left[\delta\left(\boldsymbol{k}_{a}-\boldsymbol{k}_{c}\right) \delta\left(\boldsymbol{k}_{b}-\boldsymbol{k}_{d}\right]_{\mathrm{sym}} .\right.
\end{array}
$$

(II)

$$
\sim \int \mathrm{d}^{3} \boldsymbol{k}_{1} \mathrm{~d}^{3} \boldsymbol{k}_{2} \chi_{ \pm}^{\prime}\left(\boldsymbol{k}_{a}, \boldsymbol{k}_{b} \| \boldsymbol{k}_{1}, \boldsymbol{k}_{2}\right)
$$

$\times\left[\left(\frac{\boldsymbol{k}_{1}^{2}}{2 m}+\frac{\boldsymbol{k}_{2}^{2}}{2 m}\right) \chi_{ \pm}^{*}\left(\boldsymbol{K}, m \| \boldsymbol{k}_{1}, \boldsymbol{k}_{2}\right)+\frac{1}{(2 \pi)^{3 / 2}} \int \mathrm{d}^{3} \boldsymbol{k} \tilde{V}(|\boldsymbol{k}|) \chi_{ \pm}^{*}\left(\boldsymbol{K}, m \| \boldsymbol{k}_{1}+\boldsymbol{k}, \boldsymbol{k}_{2}-\boldsymbol{k}\right)=0\right.$,

(III)

$$
\leadsto \frac{1}{2} \int \mathrm{d}^{3} \boldsymbol{k}_{1} \mathrm{~d}^{3} \boldsymbol{k}_{2} \chi_{ \pm}\left(\boldsymbol{K}_{a}, m_{\mathrm{a}} \| \boldsymbol{k}_{1}, \boldsymbol{k}_{2}\right)
$$

$$
\times\left[\left(\frac{\boldsymbol{k}_{1}^{2}}{2 m}+\frac{\boldsymbol{k}_{2}^{2}}{2 m} \chi_{ \pm}^{*}\left(\boldsymbol{K}_{b}, m_{b} \| \boldsymbol{k}_{1}, \boldsymbol{k}_{2}\right)+\frac{1}{(2 \pi)^{3 / 2}} \mathrm{~d}^{3} \boldsymbol{k} \tilde{V}(|\boldsymbol{k}|) \chi_{ \pm}^{*}\left(\boldsymbol{K}_{b}, m_{b} \| \boldsymbol{k}_{1}+\boldsymbol{k}, \boldsymbol{k}_{2}-\boldsymbol{k}\right)\right.\right.
$$

$$
=\left(\frac{\boldsymbol{K}_{b}^{2}}{4 m}-\varepsilon_{m_{b}}\right) \delta\left(\boldsymbol{K}_{a}-\boldsymbol{K}_{b}\right) \delta_{m_{a}, m_{b}}
$$


Ein Vergleich mit der Impulsdarstellung der 2-Teilchen-Schrödinger-Gleichung führt uns zu dem Lösungsansatz

$$
\begin{aligned}
& \chi_{ \pm}^{\prime}\left(\boldsymbol{k}_{1}, \boldsymbol{k}_{2} \| \boldsymbol{k}_{a}, \boldsymbol{k}_{b}=\psi_{ \pm \boldsymbol{k}_{1}, \boldsymbol{k}_{\mathbf{2}}}^{*(2)}\left(\boldsymbol{k}_{a}, \boldsymbol{k}_{b}\right),\right. \\
& \chi_{ \pm}\left(\boldsymbol{K}, m \| \boldsymbol{k}_{a}, \boldsymbol{k}_{b}\right)=\sqrt{\mathbf{2}} \psi_{ \pm \boldsymbol{k}, m}^{*(2)}\left(\boldsymbol{k}_{a}, \boldsymbol{k}_{b}\right) .
\end{aligned}
$$

Mit Verwendung der Normierungsbedingungen der Lösungen der 2-Teilchen-Schrödinger-Gleichung sehen wir, daß Gl. (I) und Gl. (III) erfüllt sind. Gl. (II) folgt aus der Orthogonalität der Streulösungen zu den 2-Teilchen-Bindungszuständen.

Mit den Ansätzen in den Gln. (2.11), (3.1) und (3.6) wird aus der Forderung Gl. (2.9) der Gültigkeit der Vertauschungsrelation Gl. (2.5)

$$
\begin{aligned}
\int \mathrm{d}^{3} \boldsymbol{k}_{1} \mathrm{~d}^{3} \boldsymbol{k}_{2} \psi_{ \pm \boldsymbol{k}_{1}, \boldsymbol{k}_{2}}^{(2)}\left(\boldsymbol{k}_{a}, \boldsymbol{k}_{b}\right) & \psi_{ \pm \boldsymbol{k}_{1}, \boldsymbol{k}_{2}}^{*}\left(\boldsymbol{k}_{c}, \boldsymbol{k}_{d}\right) \\
& +\sum_{m} \int \mathrm{d}^{3} \boldsymbol{K} \psi_{ \pm}^{(2)} \boldsymbol{K}, m
\end{aligned}
$$

Dies ist gerade die Vollständigkeitsrelation der Lösungen der 2-Teilchen-Schrödinger-Gleichung.

\section{§ 4. Zusammenhang der Transformationskoeffizienten mit den gewöhnlichen Møller- Operatoren des 2-Teilchenproblems}

Im Fall des 2-Teilchenproblems kann man zur Abbildung der freien Zustände $\varphi_{\boldsymbol{k}_{1}, \boldsymbol{k}_{2}}^{(2)}$ auf die Streuzustände $\psi_{ \pm \boldsymbol{k}_{1}, \boldsymbol{k}_{2}}^{(2)}$ gemäß

$$
\psi_{ \pm \boldsymbol{k}_{1}, \boldsymbol{k}_{2}}^{(2)}=\Omega^{(2)( \pm)} \varphi_{\boldsymbol{k}_{1}, \boldsymbol{k}_{2}}^{(2)}
$$

Møller-Operatoren $\Omega^{(2)( \pm)}\left(\mathrm{vgl}\right.$. etwa NEwTON $^{2}$ ) einführen. Diese Operatoren besitzen die Eigenschaften

$$
\begin{aligned}
\Omega^{(2)( \pm)+} \Omega^{(2)( \pm)} & =1_{\text {sym. }}^{(2)}, \\
\Omega^{(2)( \pm)} \Omega^{(2)( \pm)+} & =1_{\text {sym. }}^{(2)}-\Lambda^{(2)}, \\
\Omega^{(2)( \pm)+} \Lambda^{(2)} & =0
\end{aligned}
$$

wobei $\Lambda^{(2)}$ gemäß

$$
\Lambda^{(2)}=\sum_{m} \int \mathrm{d}^{3} \mathbf{K}\left|\psi_{\mathbf{K}, m}^{(2)}\right\rangle\left\langle\psi_{\mathbf{K}, m}^{(2)}\right|
$$

durch die Projektionsoperatoren der 2-Teilchen-Bindungszustände gegeben ist.

Außerdem gilt

$$
\Omega^{(2)( \pm)+} H^{(2)} \Omega^{(2)( \pm)}=H_{0}^{(2)} .
$$

Dies ist eine nichtlineare Bestimmungsgleichung für den Møller-Operator.

Der Møller-Operator des 2-Teilchensystems kann formal durch

$$
\Omega^{(2)( \pm)}=\int \mathrm{d}^{3} \boldsymbol{k}_{1} \mathrm{~d}^{3} \boldsymbol{k}_{2}\left|\psi_{ \pm \boldsymbol{k}_{1}, \boldsymbol{k}_{2}}^{(2)}\right\rangle\left\langle\varphi_{\boldsymbol{k}_{1}, \boldsymbol{k}_{2}}^{(2)}\right|
$$

dargestellt werden. Für die Matrixelemente des Møller-Operators folgt

$$
\left\langle\boldsymbol{k}_{a}, \boldsymbol{k}_{b}\left|\Omega^{(2)( \pm)}\right| \boldsymbol{k}_{c}, \boldsymbol{k}_{d}\right\rangle=\psi_{ \pm \boldsymbol{k} c, \boldsymbol{k} d}^{(2)}\left(\boldsymbol{k}_{a}, \boldsymbol{k}_{b}\right) .
$$

Mit Gl. (3.6) folgt damit als Zusammenhang der Transformationskoeffizienten mit den gewöhnlichen Møller-Operatoren

$$
\begin{aligned}
& \chi_{ \pm}^{\prime}\left(\boldsymbol{k}_{1}, \boldsymbol{k}_{\mathbf{2}} \| \boldsymbol{k}_{a}, \boldsymbol{k}_{b}\right)=\left\langle\boldsymbol{k}_{\mathbf{1}}, \boldsymbol{k}_{\mathbf{2}}\left|\Omega^{(2)( \pm)+}\right| \boldsymbol{k}_{a}, \boldsymbol{k}_{b}\right\rangle, \\
& \chi_{ \pm}^{\prime *}\left(\boldsymbol{k}_{\mathbf{1}}, \boldsymbol{k}_{\mathbf{2}} \| \boldsymbol{k}_{a}, \boldsymbol{k}_{b}\right)=\left\langle\boldsymbol{k}_{a}, \boldsymbol{k}_{b}\left|\Omega^{(2)( \pm)}\right| \boldsymbol{k}_{\mathbf{1}}, \boldsymbol{k}_{\mathbf{2}}\right\rangle .
\end{aligned}
$$

Setzen wir diesen Zusammenhang in Gl. (3.3) ein, so entsteht

$\left.(\mathrm{I}) \sim \int \mathrm{d}^{3} \boldsymbol{k}_{1} \mathrm{~d}^{3} \boldsymbol{k}_{\mathbf{2}} \| \boldsymbol{k}_{a}, \boldsymbol{k}_{b}\left|\Omega^{(2)( \pm)+}\right| \boldsymbol{k}_{\mathbf{1}}, \boldsymbol{k}_{\mathbf{2}}\right\rangle\left\langle\boldsymbol{k}_{\mathbf{1}}, \boldsymbol{k}_{2}\left|H^{(2)} \Omega^{(2)( \pm)}\right| \boldsymbol{k}_{c}, \boldsymbol{k}_{d}\right\rangle=\left\langle\boldsymbol{k}_{a}, \boldsymbol{k}_{b}\left|H_{0}^{(2)}\right| \boldsymbol{k}_{c}, \boldsymbol{k}_{d}\right\rangle$

2 R. G. NEwton, Scattering Theory of Waves and Particles, McGraw-Hill Book Comp., New York 1966. 
oder in formaler Darstellung

(I)

$$
\sim \Omega^{(2)( \pm)+} H^{(2)} \Omega^{(2)( \pm)}=H_{0}^{(2)} .
$$

Analog entsteht aus Gl. (3.4)

$$
\sim \int \mathrm{d}^{3} \boldsymbol{k}_{1} \mathrm{~d}^{3} \boldsymbol{k}_{\mathbf{2}}\left\langle\boldsymbol{k}_{a}, \boldsymbol{k}_{b}\left|\Omega^{(2)( \pm)+}\right| \boldsymbol{k}_{1}, \boldsymbol{k}_{\mathbf{2}}\right\rangle\left\langle\boldsymbol{k}_{1}, \boldsymbol{k}_{\mathbf{2}}\left|H^{(2)}\right| \psi_{+}^{(2)}(\boldsymbol{K}, m)\right\rangle=0
$$

oder formal

$$
\leadsto \Omega^{(2)( \pm)+} \psi_{ \pm}^{(2)} \boldsymbol{K}, m=0 .
$$

Da dies für alle $\{\boldsymbol{K}, m\}$ gilt, folgt

$$
\Omega^{(2)( \pm)+} \Lambda^{(2)}=0,
$$

wo $\Lambda^{(2)}$ der Projektionsoperator des Raumes der 2-Teilchen-Bindungszustände ist.

Die Integralgleichungen (I) und (II) reduzieren sich also auf die bekannten Bedingungen (4.6) und (4.4) der Møller-Operatoren. Die Beziehung (4.2) der Møller-Operatoren folgt direkt aus der Normierungsbedingung der Streulösungen des 2-Teilchenproblems.

Die hier gezeigten Zusammenhänge lassen sich auch expizit bei Berücksichtigung höherer Glieder der Entwicklung in Gl. (2.13) verfolgen, sie werden aber sehr verwickelt, da die Transformationskoeffizienten des $N$-Teilchenproblems mit den Møller-Operatoren aller $n$-Teilchensysteme mit $n \leqq N$ zusammenhängen.

Herrn Prof. W. WeIDLICH danke ich sehr herzlich für anregende Diskussionen und wertvolle Ratschläge. 